SU-ITP-06/13

SLAC-PUB-11846

hep-th/0605047

\title{
Attractor Explosions and Catalyzed Vacuum Decay
}

\author{
Daniel Green, Eva Silverstein, and David Starr
}

SLAC and Department of Physics, Stanford University, Stanford, CA 94305-4060

We present a mechanism for catalyzed vacuum bubble production obtained by combining moduli stabilization with a generalized attractor phenomenon in which moduli are sourced by compact objects. This leads straightforwardly to a class of examples in which the Hawking decay process for black holes unveils a bubble of a different vacuum from the ambient one, generalizing the new endpoint for Hawking evaporation discovered recently by Horowitz. Catalyzed vacuum bubble production can occur for both charged and uncharged bodies, including Schwarzschild black holes for which massive particles produced in the Hawking process can trigger vacuum decay. We briefly discuss applications of this process to the population and stability of metastable vacua.

May 2006 


\section{Introduction}

Recently much progress has been made in stabilizing moduli and analyzing the resulting discretuum of vacua [1]. Another topic of recent interest has been the attractor mechanism (see e.g. [2, 3]), in which moduli are driven to fixed values at the horizon of charged black holes.

In general, both types of effects are present. The dynamics of moduli $\phi_{i}$ are determined by the scalar potential $\mathcal{U}(\phi)$ and by other local sources $\rho(\phi ; x)$ to which the moduli couple via an equation of motion of the form

$$
\nabla^{2} \phi \sim-\partial_{\phi} \mathcal{U}(\phi)-\partial_{\phi} \rho
$$

For example, in weakly coupled string theory, the dilaton $\Phi$ modulates masses and couplings of particles, so energy densities formed from them typically depend on $\Phi$. As a specific example, moduli-dependence in the kinetic terms for electromagnetic fields yields forces on moduli near charged sources, an ingredient in the attractor mechanism.

Taking both effects into account leads to the possibility of catalyzed production of bubbles of different vacua. Consider the subset of backgrounds where structure formation leads to objects dense enough that the source terms $\rho$ in (1.1) compete with the barrier height in $\mathcal{U}$ separating one metastable minimum of $\mathcal{U}$ from another. The moduli can then be locally forced into the basin of attraction of a different minimum from that at which they are metastabilized in bulk. Depending on the parameters, this can result in vacuum decay or in production of a perturbatively stable nonexpanding bubble of a different vacuum. A dramatic string-theoretic example of this was obtained recently in [4], where certain charged sources produce a tachyonic instability nearby, catalyzing the formation of a bubble of nothing.

One goal of the present note is to point out a large class of examples where bubbles of other vacua appear in the course of Hawking evaporation of black holes. In particular, in the charged case we generalize [⿴囗十 and in the uncharged case we obtain a new mechanism for vacuum bubble production.

For charged black holes, this arises in variants of the process [4] accessible in effective field theory. One can start in one metastable vacuum and form a black hole with mass much greater than its charge. At first it behaves approximately like a Schwarzschild black hole, gradually losing mass to Hawking radiation. At some point long before it reaches the correspondence point, the black hole has radiated away enough of its mass that the charge 
plays a role, exerting local forces on the scalar fields in the problem and creating a bubble of a different vacuum.

Vacuum bubbles can also be produced in the case of uncharged compact objects, including Schwarzschild black holes, via the accumulation of uncharged matter sourcing scalar fields. Depending on the relative strength of the local energy density and the ambient moduli potential, the bubbles may appear outside or inside the Schwarzschild radius. Bubbles may form outside in two ways: in the process of collapse before the horizon forms, and in the process of Hawking evaporation as the black hole becomes small enough to produce massive Hawking particles sourcing the scalar. In the latter case, we will present a window of parameters for which this new mechanism constitutes the dominant method for producing vacuum bubbles, occurring faster than tunneling and other effects in the black hole background.

One application is to black hole physics, whose dynamics contains information about other vacua. This plays a role in the microphysical description of black holes and their information flow.

Another application is to provide a method for populating the landscape which proceeds perturbatively. Backgrounds with larger variations in density are more likely to produce vacuum bubbles; conversely backgrounds with less dense structure formation are more stable.1 The process also provides a dynamical mechanism favoring regions with light particles; this aspect is similar to moduli trapping [7] except here the particle production is effected by structure formation and evaporating black holes.

Perhaps the most compelling application of this process would be to obtain regions of parameter space where catalyzed bubble production would be possible realistically, probing regions of the landscape. As we discuss, this depends on the degree to which dense structures and small black holes form as a result of early universe inflation and structure formation. A much more extensive treatment of this appears in the interesting related work of [10] to appear. The prospect of catalyzed decays and their realistic embedding is also under investigation in [11].

1 There have been a number of calculations of catalyzed tunneling decays in field theory and gravity [5]. For simplicity we will here focus on the regime where local overdensities develop strongly enough to classically kick the scalar field over a potential barrier separating different local minima. There have been a number of works using gases of particles obtained cosmologically to provide transient forces on scalar fields, as in thermal inflation and e.g. [6, 7, 8, 9]. 
The paper is organized as follows. In section 2 we set up two canonical systems where moduli are sourced by local densities, and review bubble dynamics in a way applicable to the catalyzed formation process. The main new results are contained in section 3, where we present the catalyzed bubble production mechanisms for charged and uncharged black holes. Section 4 describes various applications of the mechanism.

\section{Catalyzed Bubble production and evolution}

Consider for simplicity first a regime in which low energy field theory is a good approximation. We will be interested in the situation described above (1.1) in which the scalar equation of motion can be written in the form

$$
\nabla^{2} \phi=-\partial_{\phi} \mathcal{U}_{t o t}(r, \phi)
$$

where the total effective potential $\mathcal{U}_{\text {tot }}$ depends on radial coordinate distance $r$ from the source, in a metric of the form

$$
d s^{2}=-a(r)^{2} d t^{2}+\frac{d r^{2}}{a(r)^{2}}+b(r)^{2} d \Omega^{2}
$$

in terms of functions $a(r), b(r)$ which approach 1 and $r$ respectively in the limit where gravitational effects are negligible.

We will find it useful to parameterize the moduli potential in terms of its energy scale $M_{U}$ and the typical size of its features in field space $M_{0}$ :

$$
\mathcal{U}=M_{U}^{4} f\left(\phi / M_{0}\right)
$$

In particular, the barrier width is of order $M_{0}$.

Let us give two illustrative examples. First, we can consider a charged compact object, with scalars $\phi_{i}$ coupling to electromagnetic fields according to the action

$$
S=\int d^{4} x \sqrt{-G}\left(M_{p}^{2} R-2\left(\partial \phi_{i}\right)^{2}-f_{a b}\left(\phi_{i}\right) F_{\mu \nu}^{a} F^{b \mu \nu}-\mathcal{U}\left(\phi_{i}\right)\right)
$$

(generalizing that in [3] to include the ambient moduli potential $\mathcal{U}$ ). For simplicity we will focus on a single direction $\phi$ scalar field space. Given spherical symmetry and Gauss' law, we can write say for a magnetically charged black hole

$$
F^{a}=Q^{a} \sin \theta d \theta \wedge d \phi
$$


The equations of motion for the scalar field in this background are

$$
\frac{1}{b^{2} \sin \theta} \partial_{\mu}\left(b^{2} \sin \theta \partial^{\mu} \phi\right)=\left(\frac{V_{e f f}^{\prime}}{2 b^{4}}+\frac{1}{4} \mathcal{U}^{\prime}\right)(\phi)
$$

where ' denotes differentiation with respect to $\phi$ and where the local contribution to the force on the scalar arises from an effective potential

$$
V_{e f f}=f_{a b}(\phi) Q^{a} Q^{b}
$$

obtained from the electromagnetic energy by plugging (2.5) into (2.4). The total effective potential in the sense of $(2.1)$ is $\mathcal{U}_{\text {tot }}=V_{\text {eff }} / b^{4}+\mathcal{U}$.

As a second illustrative example, let us consider a scalar $\phi$ which modulates the mass of a particle, for example a fermion field $\psi$. The action governing this sector of the theory is

$$
S=\int d^{4} x \sqrt{-G}\left(M_{p}^{2} R+i \bar{\psi} D \psi-2(\partial \phi)^{2}+\tilde{f}\left(\phi / \tilde{M}_{0}\right) m_{\psi} \bar{\psi} \psi-\mathcal{U}(\phi)+\Omega \bar{\psi} \gamma^{0} \psi\right)
$$

where we included the possibility of a chemical potential $\Omega$, and we parameterize the argument of the modulating function $f$ via a scale $\tilde{M}_{0}$. In a background density of $\psi$ particles, the scalar field equation of motion can again be written in the form (2.6), where in this case the local effective potential is

$$
\frac{V_{e f f}}{b^{4}}=\tilde{f}\left(\phi / \tilde{M}_{0}\right)\left\langle m_{\psi} \bar{\psi} \psi\right\rangle(r, t) \equiv \rho_{\psi}(r, t ; \phi)
$$

In a background which includes the evolution of structure, the massive fields $\psi$ will develop inhomogeneities (along with other sectors of the theory). We will be interested in cases where these inhomogeneities make the $\partial_{\phi} \rho_{\psi}$ term compete with the $\partial_{\phi} \mathcal{U}$ term in the equation of motion for $\phi$.

Having indicated two types of examples, let us return to the general analysis of the dynamics. Let us suppose that enough density develops that the forces from $V_{e f f} / b^{4}$ dominate over those of $\mathcal{U}$ for $r$ less than some crossover scale $r_{c}$. Let us start by assuming that the forces on the scalar field are dominated by field theoretic ones, i.e. that the energy densities in the two vacua are small enough that the curvature is a negligible contribution to the scalar equation of motion. We will also make use of the discretuum of vacua to assume we can tune the potential as desired to simplify our analysis. We will take the local effective potential $V_{\text {eff }}$ for simplicity to have a single minimum at $\phi=\phi_{*}$, and $\mathcal{U}$ to have a minimum at $\phi=\phi_{+}$and a lower minimum at $\phi=\phi_{-}$. In bulk, $\phi$ is metastabilized at either $\phi_{\infty}=\phi_{+}$or $\phi_{\infty}=\phi_{-}$. 

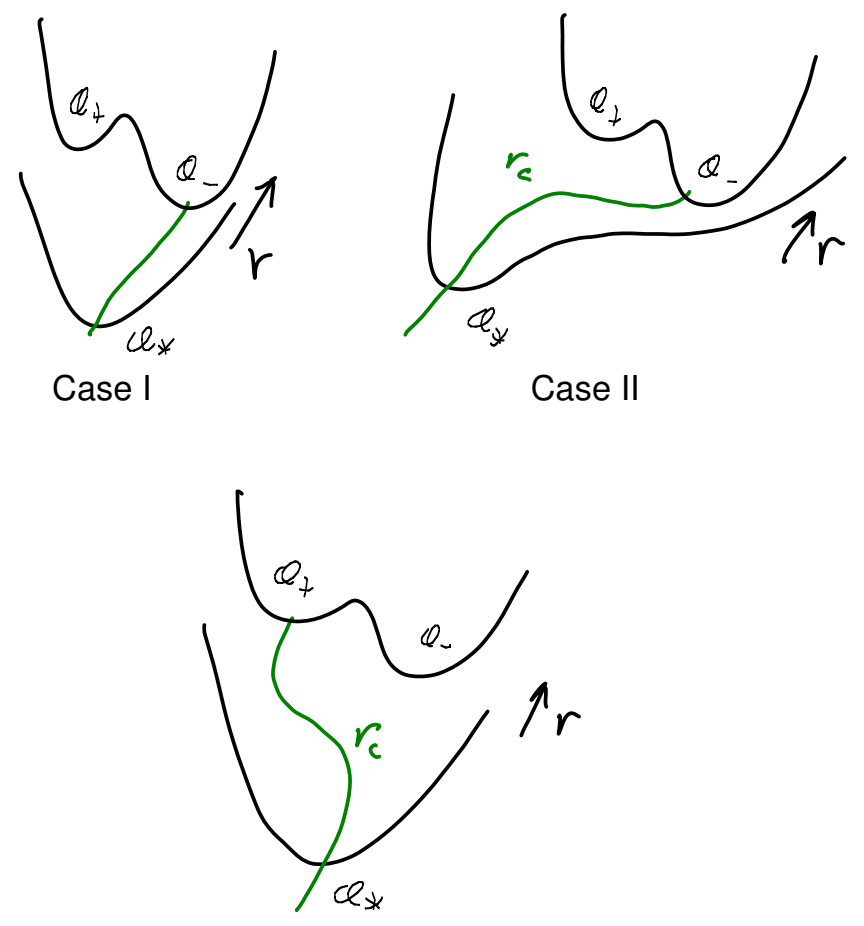

Case III

Fig. 1: Three basic cases in which a compact object locally distorts the effective moduli potential.

There are three basic cases we will discuss (see figure 1): (I) $\phi_{*}$ is in the basin of attraction of $\phi_{\infty}$, (II) $\phi_{\infty}=\phi_{-}$is in the true vacuum while $\phi_{*}$ is close to $\phi_{+}$, (III) $\phi_{\infty}=\phi_{+}$is in the false vacuum while $\phi_{*}$ is close to $\phi_{-}$. In cases II and III, one forms a vacuum bubble. This bubble is perturbatively stable at a finite radius in case II and can expand forever for appropriate values of the parameters in case III.

\subsection{Case I}

First we consider the case where $V_{\text {eff }}$ has settled down to a time independent shape, whose minimum is in the basin of attraction of $\mathcal{U}(\phi(r \rightarrow \infty))$, the minimum of $\mathcal{U}$ where $\phi$ is stabilized asymptotically. There is a static perturbatively stable solution in this case. 
At any given $r$, there exists a minimum of the combined potential $\mathcal{U}_{\text {tot }}(r, \phi)=\frac{V_{\text {eff }}}{2 b(r)^{4}}+\frac{1}{4} \mathcal{U}$ that is continuously connected to minima of $\mathcal{U}$ and $V_{\text {eff }}$.

To first approximation, $\phi$ will sit at the minimum of $\mathcal{U}_{\text {tot }}$ at each $r$ :

$$
\phi=\phi_{0}(r)+\delta \phi(x)
$$

where the background solution $\phi_{0}(r)$ satisfies

$$
\left.\frac{\delta \mathcal{U}}{\delta \phi}\right|_{\phi=\phi_{0}(r)} \equiv 0
$$

Fluctuations $\delta \phi(x)$ about the minimum are massive.

\subsection{Case II: perturbatively stable vacuum bubbles}

When $V_{\text {eff }}$ has a minimum at the false vacuum value of $\phi$, but $\phi$ is in the true vacuum asymptotically, a static bubble is formed.

There are two characteristic values of $r$ in this case. As before $r_{c}$ is the coordinate

radius below which the local effective potential $V_{e f f} / b^{4}$ draws the scalar field toward $\phi_{*}$. A second radial scale of interest, $r_{c}^{\prime}>r_{c}$ appears in this case; it is the radial distance at which the two minima of $\mathcal{U}_{\text {tot }}$ are degenerate: $\mathcal{U}_{\text {tot }}\left(\phi_{+}, r_{c}^{\prime}\right)=\mathcal{U}_{\text {tot }}\left(\phi_{-}, r_{c}^{\prime}\right)$.

For $r<r_{c}$, $\phi$ will roll toward the only minimum available. In particular, the scalar field VEV will be close to that corresponding to the false vacuum in bulk. Conversely, if this bubble of false vacuum extended out to $r \gg r_{c}^{\prime}$, then it would collapse for energetic reasons. Thus, the bubble wall should have a stable position somewhere near $r_{c}^{\prime}$.

The technology developed in [12] could be used to analyze this case in more detail; we will illustrate this in the next section on case III.

\subsection{Case III: exploding attractors and vacuum decays}

The final case is where the field is metastabilized in the false vacuum at infinity, but $V_{\text {eff }}$ is in the true vacuum. The solutions will typically evolve in time, and thus the question of initial conditions is important. Unlike the previous case, there is only one characteristic scale $r_{c}$.

We expect that for $r<r_{c}$, the field will roll into the minimum. Therefore, we will start with the bubble wall at a radius slightly below $r_{c}$ and we will consider whether or not the bubble expands. At $r \gg r_{c}, \mathcal{U}$ dominates and the solution will look like a typical vacuum bubble so the standard analysis applies to determine whether the bubble expands. 
Therefore, we will focus on the new effects of the local source. We will be interested in finding reasonable conditions under which the wall expands from its starting configuration at $r_{c}$.

Let us start in pure field theory to gain intuition, after which we will indicate the fully relativistic generalization. First let us arrange parameters such that the bubble wall is thin. This means that $\phi$ varies from $\phi_{\infty}$ to $\phi_{*}$ over a radial distance $\Delta r \ll r_{c}$. We follow [12] and work in Gaussian normal coordinates

$$
d s^{2}=\epsilon d n^{2}+\gamma_{i j} d x^{i} d x^{j}
$$

where $n$ is the normal direction to the bubble, and $x^{i}$ the coordinates along the bubble. The scalar equation of motion is then

$$
\frac{1}{2} \frac{d}{d \phi}\left(\frac{d \phi}{d n}\right)^{2}+\Gamma_{i n}^{i} \frac{d \phi}{d n}=\frac{\partial \mathcal{U}_{t o t}}{\partial \phi}
$$

A thin wall requires that $\left(\frac{d \phi}{d n}\right)^{2} \gg \Gamma_{i n}^{i} \frac{d \phi}{d n}$ at the wall. This integrates to

$$
\left(\frac{d \phi}{d n}\right)^{2}=2 \mathcal{U}_{t o t}(\phi ; \tau)+C
$$

where $\tau$ is the proper time along the wall and $C$ is an integration constant. Setting the variation of $\phi$ with respect to $n$ to be zero inside the bubble, we can take $C$ to be $-2 \mathcal{U}_{\text {tot }}\left(\phi_{*}\right)$.

Suppose the bubble starts at rest at radius just below $r_{c}$, forming once the density accumulates to the point that $V_{e f f} / b^{4}$ dominates over $\mathcal{U}$ for $r<r_{c}$. Let us expand $\mathcal{U}_{\text {tot }}\left(\phi ; r_{c}\right)=V_{0}+V_{1} \phi+\ldots$, with $V_{1}$ parameterizing the steepness of the potential which drives the field toward its inner vacuum. To begin with, the normal direction is the $r$ direction, and the equations integrate to

$$
\phi-\phi_{*}=\frac{V_{1}}{2}\left(r-r_{c}\right)^{2}
$$

If we fix the difference in field VEVs $\Delta \phi \equiv \phi_{\infty}-\phi_{*}$ and increase the strength of the potential (increase $V_{1}$ ), then we decrease the thickness $\Delta r$ of the wall and improve the thin wall approximation.

In the example of attractor black holes (2.7), this can be obtained by choosing sufficiently large charges $Q$ such that $\Delta r \ll r_{c}$. From $(2.6)(2.7)$ we obtain that the crossover 
scale $r_{c}$ occurs at $r_{c} \sim \sqrt{Q} / M_{\mathcal{U}}$ where $M_{\mathcal{U}}^{4}$ is the scale of the potential $\mathcal{U}$. So holding fixed $\Delta \phi$ and scaling up $Q$ increases $r_{c}$ and decreases the thickness, improving the thin wall approximation.

Now in order to understand if the bubble will expand, we need to compare the energy cost from the tension with the energy gained by replacing the volume inside the bubble by the phase of lower energy $\mathcal{U}\left(\phi_{*}\right)$. We will mostly analyze this with a generic potential characterized by the two scales $M_{U}, M_{0}$, in particular taking the energy difference $\mathcal{U}\left(\phi_{+}\right)-$ $\mathcal{U}\left(\phi_{-}\right) \sim M_{U}^{4}$. In a realistic example with $\mathcal{U}\left(\phi_{+}\right)$tuned to be very small, this generic energy difference applies for decays to a $\Lambda<0$ phase. A smaller energy difference corresponds to a larger critical bubble size required for expansion in the regime where $\mathcal{U}$ dominates [13].

The tension costs an amount

$$
E_{B}=4 \pi^{2} T_{B}\left(R_{B}\right) R_{B}^{2}
$$

where we have taken into account the fact that the tension will change with wall radius $R_{B}$ because our potential $\mathcal{U}_{\text {tot }}$ changes with distance from the source. In particular, the barrier between the two basins of attraction $\phi_{ \pm}$appears for large enough $R_{B}$ that $\mathcal{U}$ begins to dominate in $\mathcal{U}_{\text {tot }}$.

Plugging the solution (2.15) into the expression for the wall tension:

$$
T_{B}=\int_{\phi_{*}}^{\phi_{\infty}} d \phi \sqrt{2\left|\mathcal{U}_{t o t}(\phi)-\mathcal{U}_{t o t}\left(\phi_{\infty}\right)\right|}
$$

we obtain at $R_{B}=r_{c}$

$$
T_{B}\left(R_{B}=r_{c}\right)=\frac{2}{3} \sqrt{2 V_{1}}(\Delta \phi)^{3 / 2}
$$

Let us first compare the tension energy at $R_{B}=r_{c}$ to the potential energy $E_{b u l k}$ liberated by the bubble volume $V_{B} \sim r_{c}^{3}$. This is

$$
E_{b u l k}\left(R_{B}=r_{c}\right) \sim r_{c}^{3} V_{1} \Delta \phi \gg T_{B} r_{c}^{2}
$$

which parameterically beats the wall energy $T_{B} r_{c}^{2}$ given our thin wall parameters $r_{c} \gg \Delta r$. More generally, if we stay outside the compact source (e.g. if we stay outside the horizon of the black hole), we should consider a shell of bulk potential energy of thickness $\Delta \tilde{r}$ with volume $\Delta \tilde{r} r_{c}^{2}$ rather than the whole volume $r_{c}^{3}$. The ratio of the bulk energy to the wall energy is of order

$$
\frac{E_{b u l k}}{E_{\text {wall }}} \sim \frac{\Delta \tilde{r}}{\Delta r}
$$


There is also stress-energy from the black hole and from kinetic energy of the scalar field as it rolls toward the bottom of its local potential, and from particles that it produces in the process. Some outgoing radiation will be produced in the process of bubble formation, as well as ongoing Hawking particle production, leading to a net flux outward of energy from the compact object. As we will discuss momentarily, these effects aid the expansion of the bubble, which may play a significant role in cases where the bubble would be sub-critical from the point of view of $\mathcal{U}$ alone. First, however, let us continue to assess the expansion based on $\mathcal{U}$ in the cases where this is sufficient.

Once enough energy has escaped that the black hole horizon is separated from the wall by a distance $\Delta \tilde{r} \gg \Delta r$, the comparison (2.20) means that the wall begins to expand. The tension of the wall at larger radius increases somewhat due to the barrier in $\mathcal{U}$, but as long as the bubble size and the parameters in $\mathcal{U}$ are in the range leading to expansion according to the standard Coleman-de Luccia analysis [13], the explosion will continue unabated.

In the case of (2.8), where the local density arises from massive particles $\psi$ whose mass depends on $\phi$, the expanding bubble may produce further $\psi$ particles, amplifying the effect. This is because as $\phi$ rolls toward the new minimum $\phi_{-}$, the mass of the massive particles decreases. This can lead to quantum production of further $\psi$ particles, enhancing the local density trapping $\phi$ near $\phi_{*}$ [7].

The techniques developed in [12] provide a generally covariant description of the evolution of bubbles in a way which is convenient for incorporating the mass and charge of the black hole, and the $r$ dependence in our total effective potential $\mathcal{U}_{\text {tot }}(\phi ; r)$. The results of this section appear from such an analysis in the weak gravity limit.

As mentioned above, the inside of the bubble (and the wall itself) can contain additional sources of energy and pressure. Thus far, we have focused on the effective potential for $\phi$ while ignoring that it originates from a local source with a different equation of state from the moduli potential. Moreover, the relaxation of the $\phi$ field to its local minimum $\phi_{*}$ proceeds via transfer of its kinetic energy into particles (including further $\psi$ production as in [7]). As such, the pressure inside the wall has a different relation to the energy density than would arise from pure vacuum energy. Also, a gauge field confined to the bubble contributes a positive pressure term that would not appear if it were simply a scalar. This positive pressure inside the wall helps the bubble expand. Similar couplings to other standard model particles will also aid the expansion. 
In what follows, we will make several applications of the basic process we have discussed in this section. First we will discuss the stimulated emission of vacuum bubbles in the process of black hole formation and evaporation. The charged case $\S 3.1$ is most closely analogous to [4], but we will also find a mechanism for perturbative production of vacuum bubbles by uncharged bodies including Schwarzschild black holes $\S 3.2$. We will then move on in $\S 4$ to discuss some implications of this for the landscape, and make preliminary comments on realistic constraints.

\section{Black Holes as Catalytic Vacuum Converters}

\subsection{Vacuum bubbles as endpoints of Hawking decay: the charged case}

One application is to generalize the endpoint of Hawking decay found in [4]. Consider starting in a metastable vacuum at $\phi_{\infty}$ and forming a black hole with mass much greater than its charge. At first, the field $\phi$ is well stabilized at $\phi_{\infty}$. Once the black hole radiates down to the point that its horizon at $r=r_{h}$ falls below $r_{c}$, the local effective potential $V_{e f f} / b^{4}$ begins to pull the scalar field toward the point $\phi_{*}$ minimizing the local effective potential $V_{\text {eff }}$. In case II, this produces a metastable vacuum bubble (a vacuon). In case III, it produces an explosive vacuum decay. After the vacuum bubble is produced, the $r_{h}<r_{c}$ black hole continues to evaporate, pair producing particles in the spectrum of the inner vacuum.

Let us next compute the basic thermodynamic quantities at the point that the bubble is unleashed. This occurs when $r_{c} \sim \sqrt{Q} / M_{U}$ is of order the horizon radius $r_{h}$. For the Reissner-Nordstrom case,

$$
d s^{2}=-\left(1-\frac{2 M}{M_{P}^{2} r}+\frac{Q^{2}}{M_{P}^{2} r^{2}}\right) d t^{2}+\frac{d r^{2}}{\left(1-\frac{2 M}{M_{P}^{2} r}+\frac{Q^{2}}{M_{P}^{2} r^{2}}\right)}+r^{2} d \Omega^{2}
$$

the outer black hole horizon is at $r_{h}=\frac{M}{M_{p}^{2}}+\sqrt{\frac{M^{2}}{M_{P}^{4}}-\frac{Q^{2}}{M_{P}^{2}}}$.

When the outer horizon shrinks to $r_{c}$, the bubble emerges. As in [4], this can easily happen far away from the correspondence point, in the nonextremal regime $M / M_{P} \gg Q$. In this regime, the horizon radius is approximately $r_{h} \sim 2 M / M_{P}^{2}$. Setting this equal to $r_{c} \sim \sqrt{Q} / M_{U}$ yields $Q \sim\left(M^{2} M_{U}^{2}\right) / M_{P}^{4}$. For this to be self-consistent, $Q \ll M / M_{P}$ implies $M_{U}^{2} \ll M_{P}^{3} / M \sim M_{P} M_{U} / \sqrt{Q}$ or more simply $M_{U} \ll M_{P} / \sqrt{Q}$. Given this, the bubble is released at a point when the black hole is still very Schwarzschild-like, with temperature $T \sim M_{U} / \sqrt{Q}$ and entropy $S \sim Q M_{P}^{2} / M_{U}^{2} \gg Q^{2}$. 
It would be interesting to obtain a microphysical accounting of these objects and their explosions, a topic to which we will return briefly in the discussion section.

There are clearly many variants of this. For example, the modulus may couple to other fields which condense inside the bubble, spontaneously breaking bulk symmetries. This is a feature of the tachyon condensation in [4]. Instead of pure Reissner-Nordstrom one may consider multiple charges, such as those combinations for which a standard attractor black hole arises at the end of the process, surrounded by a vacuum bubble.

\subsection{Schwarzschild black holes and bubbles}

The process [4] and its generalization in $§ 3.1$ depend on having a charged source for the moduli. The vacuum Schwarzschild solution does not locally source scalar moduli outside the horizon. However, the moduli can be sourced by the dense matter coallescing to form the black hole. This can happen either before the horizon forms, or inside the horizon, depending on the energy scales. Moreover, in the process of Hawking decay the temperature increases to the point where massive particles get produced; as we will see these may also source moduli and yield a vacuum bubble.

\section{Bubble Catalysis in Black Hole formation I: initial collapse}

Matter which collapses to form a black hole of mass $M$ develops a density of order $M / R_{S}^{3} \sim M_{P}^{6} / M^{2}$ when the matter reaches the Schwarzschild radius $R_{S} \sim M / M_{P}^{2}$. If this density competes with the barrier heights in the moduli potential $\mathcal{U}$, and if the local and ambient potentials are configured as in case II or III above, then this process will produce a vacuum bubble before a black hole is formed. In case II, it creates a perturbatively stable vacuon, and in case III an explosive vacuum decay.

\section{Bubble Formation in Black Hole formation II: inside}

In many models, the black holes which form by structure formation have a Schwarzschild density $M_{P}^{6} / M^{2}$ which is very low compared to natural barrier heights. For example, the smallest black holes inferred from core collapse events have roughly solar mass, corresponding to an energy density of $G e V^{4}$. This means that for $\mathcal{U} \gg G e V^{4}$, no bubbles are formed outside the horizon in their formation.

However, inside the horizon, bubbles are generically classically nucleated by the crunching matter source. In particular, in weakly coupled string landscape models [1], 
there is a runaway direction for the dilaton and/or volume moduli separated by a barrier from metastable vacua. These moduli will generically couple to the masses of particles forming the black hole. As a result, a vacuum bubble containing the basin of attraction of the large volume/weak coupling limit will materialize inside the black hole. The Kasner solution approaching the black hole singularity must therefore be extended to include the rolling dilaton, volume, and other liberated moduli of the compactification inside the bubble.2

Bubble formation in Black Hole Evaporation III: massive Hawking particles seed bubbles

Let us consider a model such as (2.8) and simple generalizations in which $\phi$ modulates the mass of a set of fields such as $\psi$. In the process of evaporation, the black hole eventually reaches high enough temperatures $T$ to produce massive Hawking particles $\psi$ of mass $m_{\psi}$. Once $T$ reaches the threshold $T \sim m_{\psi}$, a significant density of the massive $\psi$ particles is produced [18]. If these particles do not decay or disperse too rapidly, they form a density of particles which can kick $\phi$ from one basin of attraction to another. We will now assess a window of parameters where this occurs. For simplicity we will first focus on a regime where the produced massive $\psi$ particles are still non-relativistic far from the $\mathrm{BH}$ (with outward velocities beating the escape velocity, but much smaller than the speed of light). The black hole also produces quanta of $\phi$ itself. For consistency we will then check that $\phi$ fluctuations do not themselves produce bubbles, and that they do not wash out the $\psi$-catalyzed bubble production in the range of parameters of interest. With $m_{\psi} \gg m_{\phi} \sim M_{U}^{2} / M_{0}$, the situation is schematically depicted in figure 2.

2 Note that this result is different from the suggestion [14] that new universes are created inside black holes, which would require the black hole singularity to be resolved in such a way as to connect a big crunch with a big bang. Evidence such as 15, 16, 17 contraindicates the hypothesis of a crunch-bang inside black holes. In any case, our results about bubble formation outside black holes support the idea that black holes induce mixing between different metastable vacua; our mechanism for producing such mixing is the much more prosaic classical catalysis effect described in the text. 


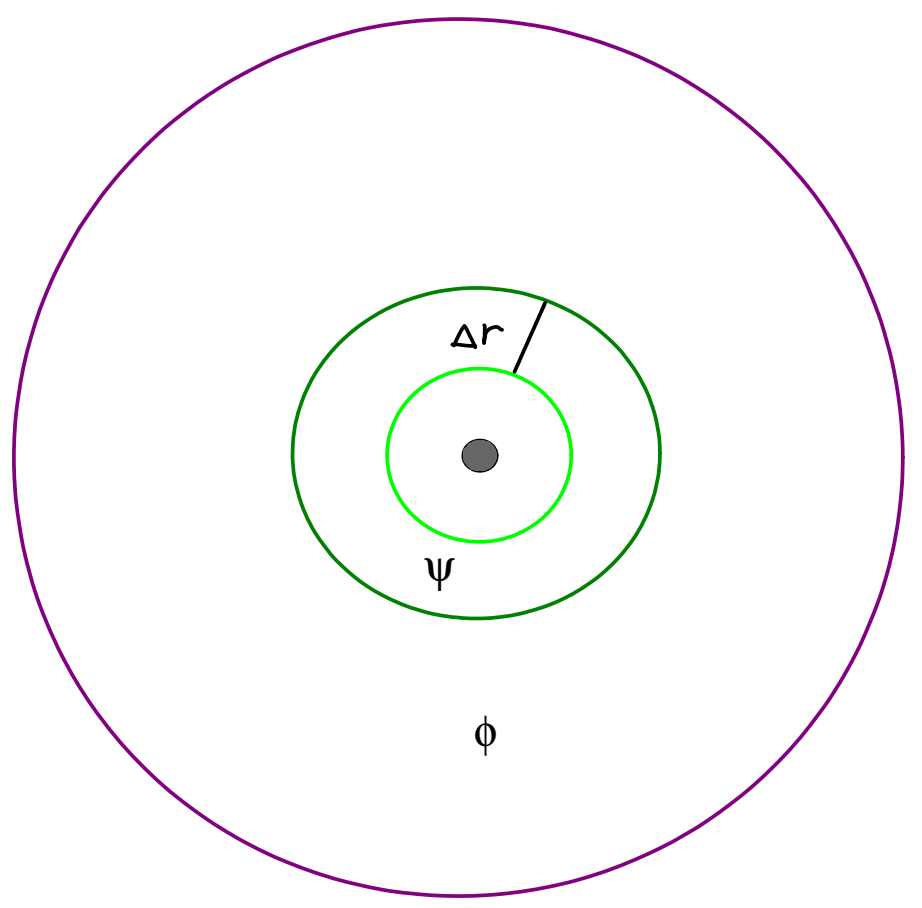

Fig. 2: In a time period $\Delta t$, the black hole (filled circle) produces a gas of massive $\psi$ particles which spreads a distance $\Delta r$ to occupy the region indicated. For the regime of parameters discussed in the text, this seeds a vacuum bubble. During the same period, the lighter $\phi$ fluctuations disperse more rapidly and have diluted to occupy a smaller energy density over a larger region. For an appropriate window of parameters the fluctuations of light $\phi$ particles do not wash out the bubble nucleated by the gas of massive $\psi$ particles.

There are five relevant scales in the problem: $M_{P}, M_{0} \sim\left|\phi_{+}-\phi_{-}\right|$(the barrier width in field space), $\tilde{M}_{0}$ (determining the $\psi-\phi$ coupling in (2.8)), $m_{\psi}$ (which will be of order the temperature $T$ in the regime of interest), and $M_{U}$ (the energy scale in the potential $\left.\mathcal{U} \sim M_{U}^{4} f\left(\phi / M_{0}\right)\right)$.

We will consider moduli for which $M_{U} \ll M_{0}$. This yields weak self-interactions in $\mathcal{U}$. The couplings between $\psi$ and $\phi$ go like $m_{\psi} / \tilde{M}_{0}(2.8)$. For the catalysis effect we will 
be led to a window in which $m_{\psi} \geq M_{0}$, requiring us to take $\tilde{M}_{0}>M_{0}$ to avoid $\geq \mathcal{O}(1)$ interactions between $\psi$ and $\phi$. In order to avoid large renormalizations of $\mathcal{U}$ due to these couplings, one can also consider a supersymmetrized version of the model with a SUSY breaking scale much lower than $M_{0}$.

The energy density in $\psi$ particles is of order

$$
\rho_{\psi} \sim \frac{\Delta M \xi}{(V o l)}
$$

where $V o l$ is the volume of space into which the produced $\psi$ particles have dispersed in a time window for which the black hole mass has decreased by $\Delta M$, of which a fraction $\xi$ has gone into the production of $\psi$ particles. In this time window $\Delta t$, the particles spread out a distance $\Delta r$, which we will insist be much greater than the black hole size $T^{-1}$. This ensures that the particles we consider are far from the black hole horizon, so that the Hawking calculation of the asymptotic produced particle distribution is accurate. More specifically, let us count the particles emerging from the black hole starting at a distance $\Delta r \gg 1 / T$, and consider their further spread into the volume contained between $r=\Delta r$ and $r=2 \Delta r$.

We will insist that $\Delta r$ be at least as large as the critical bubble size $R_{c} \sim M_{0} / M_{U}^{2}$ above which the bubble expands in the regime where the potential $\mathcal{U}$ dominates (as derived in the standard analysis [13] and reviewed in $\S 2$ ). Given $\Delta r \gg T^{-1}$, the volume $V o l$ in (3.2) is of order $\Delta r^{3}$. We will find conditions under which the density (3.2) is competitive with (or stronger than) the ambient potential $\mathcal{U}$, and that the time window $\Delta t$ is sufficiently long that the local density (3.2) can drive $\phi$ across the barrier, a distance $M_{0}$ in field space.

As the black hole temperature $T$ increases to the threshold $T=m_{\psi}$ to produce $\psi$ 's, at first it produces them non-relativistically. Their velocity is then of order

$$
v=\sqrt{2\left(\frac{E_{\psi}}{m_{\psi}}-1\right)}
$$

using $\frac{1}{2} m_{\psi} v^{2}=E_{\psi}-m_{\psi}$. As long as the massive particles are non-relativistic, their velocity remains small and their dispersal is correspondingly slow allowing them to form a dense source for $\phi$ (as we will see in what follows). The non-relativistic approximation remains valid as long as we consider a period in which $T$ remains close enough to $m_{\psi}$ that $E_{\psi} \sim T$ satisfies

$$
\frac{E_{\psi}-m_{\psi}}{m_{\psi}} \equiv \frac{\Delta T}{T} \ll 1
$$


From this we can obtain the fraction $\xi$ of the black hole emission which is contained in non-relativistic $\psi$ particles, as follows. Hawking evaporation produces a thermal distribution of particle numbers [18], up to the greybody factor arising from the absorption cross section $\sigma(E)$. For a given species, the number $N$ of particles produced by the black hole as a function of time is given by

$$
\frac{d N}{d t}=\frac{v \sigma(E)}{e^{E / T} \pm 1} \frac{d^{3} \vec{k}}{(2 \pi)^{3}}=\frac{\sigma(E)}{e^{E / T} \pm 1} \frac{v^{2} E^{2} d E}{2 \pi^{2}}
$$

where $\sigma(E)$ is of order $1 /\left(T^{2} v^{2}\right)$ [19].

For a nonrelativistic species such as our $\psi$ particles, integrating this from $E=m_{\psi}$ to $E \sim T=m_{\psi}+\frac{1}{2} m_{\psi} v^{2}$ yields $d N / d t$ of order $(v \sigma(E)) v^{3} m_{\psi}^{3} \sim v^{2} m_{\psi}^{3} / T^{2}$. For a relativistic species, integrating it from $E=0$ to $E \sim T$ gives a result for $d N / d t$ of order $\sigma(E) m_{\psi}^{3} \sim$ $m_{\psi}^{3} / T^{2}$

So the non-relativistic emission of $\psi$ particles is down by a factor of order

$$
\xi \sim v^{2} \sim \frac{\Delta T}{T}
$$

from the total emission of the black hole in this window.

To obtain the other factors in $\rho_{\psi}(3.2)$, we must relate $\Delta M$ and $\Delta r \sim(V o l)^{1 / 3}$ to $\Delta T / T$. This goes as follows. The change in mass is

$$
\Delta M \sim M_{p}^{2}\left(\frac{1}{m_{\psi}}-\frac{1}{m_{\psi}+\Delta T}\right) \sim \frac{M_{p}^{2}}{T}\left(\frac{\Delta T}{T}\right)
$$

As discussed above, we will consider a window such that $\Delta r$ is at least as big as the critical bubble size $R_{c} \sim M_{0} / M_{U}^{2}$ in the potential $\mathcal{U}$. So set

$$
\Delta r=\eta M_{0} / M_{U}^{2} \sim v \Delta t \sim \Delta t \sqrt{\Delta T / T}
$$

with $\eta \geq 1$, and relate $\Delta t$ to $\Delta T$ as follows. The window of temperatures in the range $\left(m_{\psi}, m_{\psi}+\Delta T\right)$ corresponds to the time period

$$
\Delta t \sim \frac{M_{p}^{2}}{3}\left(\frac{1}{T^{3}}-\frac{1}{(T+\Delta T)^{3}}\right) \sim\left(\frac{\Delta T}{T}\right) \frac{M_{p}^{2}}{T^{3}}
$$

obtained by integrating the Stefan-Boltzmann law $d M / d t \sim($ area $) \times T^{4} \sim T^{2}$ using $M \sim M_{P}^{2} / T$. 
Plugging (3.9) into (3.8), we obtain

$$
v^{3} \sim\left(\frac{\Delta T}{T}\right)^{3 / 2} \sim \frac{T^{3} M_{0} \eta}{M_{P}^{2} M_{U}^{2}} \ll 1
$$

with the last inequality enforced for self-consistency of the non-relativistic approximation.

Putting these estimates together, we obtain

$$
\rho_{\psi} \sim M_{U}^{4} \frac{v T^{2}}{\eta^{2} M_{0}^{2}}
$$

We will shortly impose the condition that this density not decay rapidly by annihilation into $\phi$ particles. First let us proceed to analyze its effects given this.

In order for the forces on $\phi$ from this energy density $\rho_{\psi}$ to compete with the ambient potential $\mathcal{U} \sim M_{U}^{4} f\left(\phi / M_{0}\right)$, we require $\left(\partial_{\phi} \rho_{\psi} \sim \rho_{\psi} / \tilde{M}_{0}\right)>\left(\partial_{\phi} \mathcal{U} \sim M_{U}^{4} / M_{0}\right)$ which translates into the statement

$$
v T^{2} \geq \eta^{2} M_{0} \tilde{M}_{0}
$$

This is consistent with our condition for perturbativity, $\tilde{M}_{0} \geq\left(T \sim m_{\psi}\right) \geq M_{0}$.

From (2.8) we see that the dimensionless couplings between $\psi$ and $\phi$ are of order $m_{\psi} / \tilde{M}_{0}$. Since $T \sim m_{\psi}$, if we took $\tilde{M}_{0} \sim M_{0}$, (3.12) would lead to $\geq \mathcal{O}(1)$ couplings between $\phi$ and $\psi$. In order to avoid that, we may specify that $\phi$ couples to $\psi$ via weaker (e.g. $\tilde{M}_{0} \sim M_{P}$-suppressed) couplings than appear in $\mathcal{U}$.

It is also important to check that the mean field treatment of the $\psi$ energy density is appropriate. That is, we must check that the spacing $L$ between $\psi$ particles is less than the Compton wavelength $m_{\phi}^{-1} \sim M_{0} / M_{U}^{2}$ of $\phi$. For the minimal density that competes with the moduli potential, number density of $\psi$ particles is of order

$$
n_{\psi} \sim \frac{\rho_{\psi}}{m_{\psi}} \sim \frac{\rho_{\psi}}{T} \sim \frac{M_{U}^{4} \tilde{M}_{0}}{T M_{0}} \sim \frac{1}{L^{3}}
$$

Setting $1 / L^{3} \gg m_{\phi}^{3}$ translates into the requirement

$$
\left(M_{U} / M_{0}\right)^{2}\left(T / \tilde{M}_{0}\right) \ll 1
$$

We must also check that the time window $\Delta t$ (3.9) is sufficiently long that the density $\rho_{\psi}$ has time to kick $\phi$ across the barrier. We can relate $\Delta t$ to the range of field space 
$\Delta \phi$ that $\phi$ rolls during the process as follows. As before let us expand the potential $\mathcal{U}_{\text {tot }} \sim V_{0}+V_{1} \phi+\ldots$. In a local region, the scalar rolls according to $\partial_{t}^{2} \phi \sim V_{1}$ i.e.

$$
\Delta \phi \sim V_{1}(\Delta t)^{2}
$$

In the case that the accumulated $\psi$ density is of order $M_{U}^{4}$ (and hence competitive with the ambient moduli potential), we can identify $V_{1} \geq M_{U}^{4} / M_{0}$ yielding

$$
\Delta \phi \geq \frac{M_{U}^{4}}{M_{0}}(\Delta t)^{2} \sim M_{0} \eta^{2} \frac{T}{\Delta T} .
$$

which is automatically greater than $M_{0}$ in our non-relativistic regime. So the window $\Delta T$ is easily long enough to drive $\phi$ into the basin of attraction of a different minimum.

Now let us address the decay of the density (3.11) via annihilation of $\psi$ particles into $\phi$ particles. As we noted above, a natural model in which to apply this would be a low energy supersymmetric model, for which $\psi$ has scalar superpartners also with mass of order $m_{\psi}$. The $2 \rightarrow 2$ center of mass scattering cross section $\sigma$ for scalars scales like

$$
\sigma \sim \lambda^{2} \frac{k_{f}}{k_{i}} \frac{1}{4 \pi E^{2}}
$$

where $\lambda \sim \frac{m_{\psi}^{2}}{\tilde{M}_{0}^{2}}$ is the quartic coupling, $k_{f}$ is the final momentum (of order $m_{\psi}$ in our problem), and $k_{i}$ is the initial momentum scale (of order $v m_{\psi}$ in our case). Let us choose $\tilde{M}_{0}$ large enough that this is $\leq 1$, i.e. $\tilde{M}_{0} \geq m_{\psi}$.

Given the $\psi$ density $n_{\psi} \sim \rho_{\psi} / m_{\psi}$ (3.11), velocity $v$ (3.3)(3.4), and cross section $\sigma$ (3.17), the annihilation rate for a given $\psi$ particle is

$$
\Gamma=n_{\psi} v \sigma
$$

Setting

$$
\Gamma \Delta t \ll 1
$$

ensures that the $\psi$ particles do not typically annihilate during our window of interest. In terms of our parameters, this is the condition

$$
\frac{v^{3} M_{P}^{2} M_{U}^{4}}{\eta^{2} \tilde{M}_{0}^{4} M_{0}^{2}} \ll 1
$$

The fermion annihilation cross section is down from the scalar one (3.17) by a factor of $p_{i} / m_{\psi} \sim v \ll 1$. 
Finally let us check the effects of $\phi$ fluctuations themselves. First, let us note that the gases of particles produced by the black hole (including both $\phi$ fluctuations and the $\psi$ particles in our model) are not in thermal equilibrium at temperature $T$ in the regime of interest. The particles are produced by the hot black hole, but spread out to radii of order $\Delta r \gg 1 / T$ and hence their energy density is not that of an equilibrium thermal gas at temperature $T$.

We want to check if the $\phi$ particles produce a substantial back reaction on the system. We will address the effects of the $\phi$ fluctuations in both the non-relativistic and relativistic regimes.

Expanding our potential $\mathcal{U} \sim M_{U}^{4} f\left(\phi / M_{0}\right)$ we have a $\phi$ mass $m_{\phi} \sim M_{U}^{2} / M_{0}$. As the black hole heats up past the threshold $T_{\phi} \sim M_{U}^{2} / M_{0}$ for producing $\phi$ perturbations, it begins to do so non-relativistically similarly to our discussion above. However there we saw (3.12) that $T \gg M_{0}$ was required to catalyze a bubble of radius greater than the critical size (assuming the effective potential in the presence of the gas of particles drives $\phi$ in this direction). So for $M_{U}<M_{0}$, as happens for weakly coupled moduli, the regime where non-relativistic $\phi$ particles are produced does not in itself produce a $\Delta r$-sized bubble.

In the regime $T \gg m_{\phi}$ in which the $\phi$ fluctuations are produced relativistically, the typical momentum of produced $\phi$ particles is of order $k \sim T \gg M_{U}^{2} / M_{0}$. The kinetic energy $k^{2} \phi^{2}$ is much greater than the potential energy $m_{\phi}^{2} \phi^{2}$. Similarly to the discussion above, we can estimate the energy density $\rho_{\delta \phi}$ in relativistic $\phi$ particles at a radial distance of order the critical radius $R_{c} \sim M_{0} / M_{U}^{2}$. This yields $\rho_{\delta \phi} \sim M_{U}^{4}\left(T / M_{0}\right)^{2}$. The force on the field comes only from the potential energy component of this, which is of order $\rho_{\delta \phi}\left(m_{\phi}^{2} / k^{2}\right) \sim M_{U}^{4}\left(M_{U} / M_{0}\right)^{4} \ll M_{U}^{4}$. So the effective force in the presence of the $\phi$ particles in the relativistic regime is also too weak to kick the field across the potential barrier.

As pointed out in [11], the suppression factor $m_{\psi}^{2} / k^{2}$ in the above estimate would be weakened if the energy scale $k$ were much lower than the black hole temperature $T$. This may happen via thermalization in the presence of sufficiently strong interactions among the $\delta \phi$ particles. But in the weakly coupled regime $M_{U} \ll M_{0}$ we consider here we find this effect is not significant.

This result is perhaps not surprising, given that it has been argued that symmetry is not restored in the thermal bath seen by a non-inertial observer [20]. The usual explanation for this result is that the acceleration and the temperature scale in the same way, so the effective gravity spoils the symmetry restoration that occurs in flat space. What makes 
the transition possible in the case of the $\psi$ particles is precisely that a significant density is formed at distances large enough to ignore gravitational effects. The fact that we cannot achieve this with the weakly interacting $\phi$ particles may not be a coincidence.

So far we have seen that $\phi$ fluctuations do not themselves produce large vacuum bubbles; this means the effect we are considering does require the extra $\psi$ particles. Next let us check whether the kinetic-energy-dominated spatially varying $\phi$ fluctuations wash out the bubble produced by the $\psi$ gas. The average field fluctuation $\delta \phi$ is determined by $k^{2} \delta \phi^{2} \sim \rho_{\delta \phi}$, with $k \sim T$. This could potentially be a problem if the distance in field space $\delta \phi \sim \sqrt{\rho_{\delta \phi}} / T$ is greater than or equal to the barrier width $M_{0}$. In the same window considered above for $\psi$-catalyzed bubble production, the density in $\phi$ particles is of order

$$
\rho_{\delta \phi} \sim M_{U}^{4} \frac{T^{2}}{\eta^{2} M_{0}^{2}}
$$

Setting this less than $M_{0}^{2} T^{2}$ ensures that $\delta \phi<M_{0}$ so that the $\phi$ fluctuations do not wash out the $\psi$ catalysis effect. This is automatic for $M_{U}<M_{0}$.

In the regime of parameters we have taken the $\psi$ catalysis occurs classically once the $\psi$ particles have been created, so this effect automatically dominates over exponentially suppressed thermal and tunneling effects.

It is readily verified that a window of parameters exists where all the above constraints are satisfied. As a specific example, the following hierarchy of scales works:

$$
\eta=10 \quad \frac{M_{0}}{M_{U}} \sim 10^{2} \quad \frac{m_{\psi}}{M_{U}} \sim 10^{8} \quad \frac{\tilde{M}_{0}}{M_{U}} \sim 10^{9} \quad \frac{M_{P}}{M_{U}} \sim 10^{16}
$$

Altogether these estimates suggest that vacuum bubbles can also appear as new endpoints of Hawking radiation in the case of uncharged black holes! Whatever formed the black hole, the process of evaporation proceeds through higher and higher temperatures, eventually producing massive particles which can seed a vacuum bubble surrounding the evaporating black hole if the mass scales lie in the range satisfying the above constraints.

The above estimates are in fact somewhat more conservative than necessary. For example, it is possible to extend this mechanism to the regime where the produced $\psi$ particles are relativistic. It is only the $m_{\psi} \bar{\psi} \psi$ contribution to the stress energy which sources the field, which is down by a factor of $\left(m_{\psi} / T\right)^{2}$ from the full energy density in the relativistic regime. But the production of relativistic $\psi$ particles is unsuppressed. Altogether, requiring the force at $r \sim \eta R_{c}$ to be greater than that from $\mathcal{U}$ leads to a 
somewhat wider window of parameters where the effect occurs. For example, the constraint (3.12) becomes

$$
m_{\psi}^{2} \geq \eta^{2} M_{0} \tilde{M}_{0}
$$

Moreover once the bubble is produced (either relativistically or non-relativistically), further $\psi$ particles - produced from the black hole and from the rolling $\phi$ field - push it out further.

\section{Other applications}

\subsection{Population and stability of the landscape}

We have just found a significant range of parameters for which Schwarzschild black holes ultimately catalyze vacuum bubbles in the process of Hawking evaporation if not before, since they produce massive particle densities that source the dilaton runaway direction. The decay of large black holes, while a very long process, is parameterically faster than bubble nucleation by tunneling.

This effect must be taken into account in assessing stability of metastable vacua. Realistic application requires tuning the cosmological constant to be small, and then comparing the particle spectrum and parameters in the potential to those required above for catalyzed decay. Transitions from a realistically small cosmological constant to a $\Lambda=0$ minimum involve a very small bulk potential energy difference, which makes it more difficult to obtain an explosive decay. However transitions from realistic cosmological constant vacua to nearby $\Lambda<0$ phases are not so suppressed, and the above mechanism can destabilize the model well before tunneling events do.

In the context of the landscape, this appears to be the dominant instability in a significant range of backgrounds. For example, in the case of our universe with solar mass black holes formed from core collapse events, the decay time arising from the evaporation of these black holes, of order $\sim 10^{65}$ years, is substantially shorter than the decay time of order $e^{10^{120}}$ obtained from tunneling in appropriate regions of parameter space [1] (though still safely longer than the age of the universe). This alone, while leading to a vastly shorter decay timescale, does not constrain the models realistically. However in the context of models leading to denser objects [10,21], there may be phenomenologically significant constraints from this process [10].

One can also apply this to the early universe as a mechanism for populating the landscape. Backgrounds in which overdensities develop which compete with the barriers in 
$\mathcal{U}$ will experience catalyzed vacuum bubble production. The formation of dense structures is a somewhat delicate process; in the observable universe the formation of dense stars inside gravitational potential wells induced by dark matter depends on appropriate cooling mechanisms (which may then collect yet denser structures [10,21]). In any case, in the apparently vast discretuum of string vacua, there may be many examples with dense structures forming in hidden sectors, which lead to vacuum bubble production. In the case (III) of explosive vacuum decay, this process populates the landscape faster than occurs via tunneling.

This produces a dynamical trend toward solutions with smaller inhomogeneities: backgrounds with large inhomogeneities seed vacuum decays, producing new backgrounds which produce their own bubbles until the process shuts off with the production of backgrounds with small inhomogeneities relative to barrier heights.

This dynamics also produces bubbles with lower mass particles, since the forces that draw $\phi$ toward the new vacuum at $\phi_{*}$ arise by virtue of particles whose mass decreases as $\phi \rightarrow \phi_{*}$. Although our mechanism here is different, the same trend as in [7] toward points with extra light particles arises in this context. Moreover, the bubble need not be empty; as the bubble expands and the field rolls toward $\phi_{*}$, the masses of the $\psi$ particles change with time and they may get produced.

\subsection{Vacuum bubble production in realistic models: constraints and corners}

In a highly model-dependent way, vacuum bubbles may be produced by catalysis in realistic scenarios. This depends on the densest structures that form in the model.

Some inflation models such as certain hybrid inflation models [22] produce small primordial black holes. In the context of the landscape, their formation and decays can produce vacuum bubbles instead of simply leaving behind small bursts of radiation. For case III this constrains inflationary parameter space to some extent, and conversely in case II it provides a model-dependent mechanism for producing vacuum bubbles.

A more interesting regime where dense structures may form [10] is via the collection and coalescence of charged exotics in stars, generalizing the effect discussed in [21.

Another natural question is whether real world core collapse events can produce transient vacuum bubbles; in general it is of interest to explain type II supernova explosions 23. 3. Scalar field moduli typically couple to standard model fields. These may be stabilized at a very high scale, suppressing catalyzed decays. However, very low potentials

3 Heterotic supernovae have yet to be detected. 
can be obtained technically naturally, for example in the case that a scalar field $\phi$ couples preferentially to the neutrino mass term (cf [24]) in a similar way at low energies to the example (2.8). In this case, one obtains a potential energy of order $m_{\nu}^{2} M_{C}^{2}$ where $M_{C}$ is the effective cutoff in neutrino loop contributions to the moduli potential. If the latter is at the supersymmetry breaking scale of $T e V$, the resulting energy scale for the radiatively generated moduli potential is roughly of order $M e V^{4}$, i.e. $M_{U} \sim M e V$.

The densest known structures are the neutron stars formed from core collapse events. The energy density in these environments is of order the QCD scale; the neutrinos form a trapped degenerate relativistic gas with chemical potential of order $200 \mathrm{MeV}$ and hence energy density $(200 \mathrm{MeV})^{4}$ in the core [25]. However, the scalar fields modulating the neutrino masses are directly sensitive only to the nonrelativistic correction to the energy density. This is down by a factor of order $m_{\nu}^{2} / p^{2} \sim 10^{-18}$ for momentum scale $p \sim 200 M e V$ from the relativistic energy $(200 \mathrm{MeV})^{4}$.

Hence absent further cancellations, the mass-dependent contribution to the energy density (to which the scalar is directly sensitive) is small compared to the natural scale of barrier heights. However, in models with low barrier heights, attractor explosions might play a role.

\section{Discussion}

In this note, we have seen how starting in a single metastable vacuum, one can assemble compact objects which unveil bubbles of other vacua. We found large classes of examples generalizing [4] to provide vacuum bubbles as endpoints of Hawking evaporation of charged and uncharged black holes. We have also noted that matter sources can produce bubbles of other vacua inside the horizon, which modify the internal solution before impinging on the singularity; their existence must be imprinted in the decay products of the black hole.

Perhaps most interestingly, we found that in the process of evaporation of Schwarzschild black holes, the massive particles produced in the Hawking process can seed vacuum decay.

4 The present simulations aiming to explain type II supernovae via neutrino energy deposition do not consistently yield explosions [23], so it is conceivable that new physics will be required. However it is entirely possible that explosions will arise from more conventional mechanisms (see e.g. [26]), so this rather tuned regime of parameters does not appear well motivated unless serious puzzles with generating SNe explosions persist. 
The black hole catalytically converts whatever formed it into all the particles of the system, including massive particles sourcing moduli. The resulting local potential forces the moduli into a different vacuum for a range of model parameters. This perturbative effect occurs much more rapidly than exponentially suppressed thermal and tunneling effects.

Our analysis has been semiclassical, combining structure formation and gravitational collapse with Hawking radiation and classical bubble nucleation. The basic laws of black hole mechanics [27] are classical, but provided clues pointing toward a more microphysical statistical description of black holes. It would be interesting to determine a dual microphysical description of these processes.

In general, our main lesson is simple. Compact objects made in one vacuum contain information about other vacua, if the objects are sufficiently dense. This regime seems potentially more accessible than non-perturbative cosmological methods for connecting present physics with the other vacua in the landscape. Moreover dual descriptions of black hole entropy and dynamics must account for attractor explosions in the generic setting [1]. Finally, assessing the ultimate decay modes of physical models built on metastable vacua requires analyzing the structure formation in the models and estimating the catalyzed decay time as well as the ambient tunneling amplitude.

\section{Acknowledgements}

We would like to thank T. Banks, M. Douglas, G. Horowitz, S. Kachru, R. Kallosh, A. Linde, J. Polchinski, S. Shenker, and J. Wacker for useful discussions and N. Arkani-Hamed, S. Dimopoulos, P. Schuster, and N. Toro additionally for sharing their insights into vacuon and black hole formation mechanisms [10]. We thank P. Schuster for extensive discussions on the process of $\S 3.2$, including identifying a missing factor of $v$. We are supported in part by the DOE under contract DE-AC03-76SF00515 and by the NSF under contract 9870115 . 


\section{References}

[1] R. Bousso and J. Polchinski, "Quantization of four-form fluxes and dynamical neutralization of the cosmological constant," JHEP 0006, 006 (2000) [arXiv:hep-th/0004134]; S. B. Giddings, S. Kachru and J. Polchinski, "Hierarchies from fluxes in string compactifications," Phys. Rev. D 66, 106006 (2002) [arXiv:hep-th/0105097]; E. Silverstein, "(A)dS backgrounds from asymmetric orientifolds," Contributed to Strings 2001, [arXiv:hep-th/0106209]; A. Maloney, E. Silverstein and A. Strominger, "De Sitter space in noncritical string theory," Published in *Cambridge 2002, The future of theoretical physics and cosmology* 570-591, [arXiv:hep-th/0205316]; B. S. Acharya, "A moduli fixing mechanism in M theory," arXiv:hep-th/0212294. S. Kachru, R. Kallosh, A. Linde and S. P. Trivedi, Phys. Rev. D 68, 046005 (2003) [arXiv:hep-th/0301240]. et seq.

[2] S. Ferrara, R. Kallosh and A. Strominger, "N=2 extremal black holes," Phys. Rev. D 52, 5412 (1995) [arXiv:hep-th/9508072].

[3] K. Goldstein, N. Iizuka, R. P. Jena and S. P. Trivedi, "Non-supersymmetric attractors," Phys. Rev. D 72, 124021 (2005) [arXiv:hep-th/0507096].

[4] G. T. Horowitz, "Tachyon condensation and black strings," JHEP 0508, 091 (2005) [arXiv:hep-th/0506166]; S. F. Ross, "Winding tachyons in asymptotically supersymmetric black strings," JHEP 0510, 112 (2005) [arXiv:hep-th/0509066].

[5] I. K. Affleck and F. De Luccia, "Induced Vacuum Decay," Phys. Rev. D 20, 3168 (1979). M. B. Voloshin, "Catalyzed decay of false vacuum in four-dimensions," Phys. Rev. D 49, 2014 (1994). V. A. Rubakov and D. T. Son, "Instanton like transitions at high-energies in $(1+1)$-dimensional scalar models. 2. Classically allowed induced vacuum decay," Nucl. Phys. B 424, 55 (1994) [arXiv:hep-ph/9401257]. V. A. Berezin, V. A. Kuzmin and I. I. Tkachev, "Black Holes Initiate False Vacuum Decay," Phys. Rev. D 43, 3112 (1991); A. Gomberoff, M. Henneaux, C. Teitelboim and F. Wilczek, "Thermal decay of the cosmological constant into black holes," Phys. Rev. D 69, 083520 (2004) [arXiv:hep-th/0311011].

[6] T. Damour and A. M. Polyakov, "The String dilaton and a least coupling principle," Nucl. Phys. B 423, 532 (1994) [arXiv:hep-th/9401069].

[7] L. Kofman, A. Linde, X. Liu, A. Maloney, L. McAllister and E. Silverstein, "Beauty is attractive: Moduli trapping at enhanced symmetry points," JHEP 0405, 030 (2004) [arXiv:hep-th/0403001].

[8] N. Kaloper, J. Rahmfeld and L. Sorbo, "Moduli entrapment with primordial black holes," Phys. Lett. B 606, 234 (2005) [arXiv:hep-th/0409226].

[9] T. Battefeld and S. Watson, "String gas cosmology," arXiv:hep-th/0510022.

[10] N. Arkani-Hamed, P. Schuster, N. Toro, "Observational Constraints on the Vacuum of our Universe from Catalyzed Vacuum Decay", to appear. 
[11] A. Aguirre, T. Banks, M. Dine, M. Johnson, A. Shomer, work in progress

[12] V.A. Berezin, V.A. Kuzmin and I.I. Tkachev, "Dynamics of Bubbles in General Relativity", Phys. Rev. D 36, 2919

[13] S. R. Coleman and F. De Luccia, "Gravitational Effects On And Of Vacuum Decay," Phys. Rev. D 21, 3305 (1980).

[14] L. Smolin, "Cosmological natural selection as the explanation for the complexity of the universe," PhysicaA 340, 705 (2004); E. J. Martinec, "Space - like singularities and string theory," Class. Quant. Grav. 12, 941 (1995) [arXiv:hep-th/9412074].

[15] G. T. Horowitz and J. Polchinski, "Instability of spacelike and null orbifold singularities," Phys. Rev. D 66, 103512 (2002) [arXiv:hep-th/0206228].

[16] J. McGreevy and E. Silverstein, "The tachyon at the end of the universe," JHEP 0508, 090 (2005) [arXiv:hep-th/0506130].

[17] G. T. Horowitz and J. Maldacena, "The black hole final state," JHEP 0402, 008 (2004) [arXiv:hep-th/0310281]; G. T. Horowitz and E. Silverstein, "The inside story: Quasilocal tachyons and black holes," Phys. Rev. D 73, 064016 (2006) [arXiv: hepth/0601032].

[18] S. W. Hawking, "Black Hole Explosions," Nature 248, 30 (1974). S. W. Hawking, "Particle Creation By Black Holes," Commun. Math. Phys. 43, 199 (1975) [Erratumibid. 46, 206 (1976)]. D. N. Page, "Particle Emission Rates From A Black Hole. III. Charged Leptons From A Nonrotating Hole," Phys. Rev. D 16, 2402 (1977);

[19] W. G. Unruh, "Absorption Cross-Section Of Small Black Holes," Phys. Rev. D 14, 3251 (1976).

[20] W. G. Unruh and N. Weiss, "Acceleration radiation in interacting field theories," Phys. Rev. D 29, 1656 (1984);

[21] A. Gould, B. T. Draine, R. W. Romani and S. Nussinov, "Neutron Stars: Graveyard Of Charged Dark Matter," Phys. Lett. B 238, 337 (1990).

[22] A. M. Green and A. R. Liddle, "Constraints on the density perturbation spectrum from primordial black holes," Phys. Rev. D 56, 6166 (1997) [arXiv:astro-ph/9704251]. J. Garcia-Bellido, A. D. Linde and D. Wands, "Density perturbations and black hole formation in hybrid inflation," Phys. Rev. D 54, 6040 (1996) [arXiv:astro-ph/9605094].

[23] S. Woosley and T. Janka, "The Physics of Core-Collapse Supernovae," arXiv:astroph/0601261.

[24] R. Fardon, A. E. Nelson and N. Weiner, "Dark energy from mass varying neutrinos," JCAP 0410, 005 (2004) [arXiv:astro-ph/0309800].

[25] G. G. Raffelt, "Stars as laboratories for fundamental physics: The astrophysics of neutrinos, axions, and other weakly interacting particles " Chicago, USA: Univ. Pr. (1996) $664 \mathrm{p}$.

[26] A. Burrows, E. Livne, L. Dessart, C. Ott and J. Murphy, "A New Mechanism for Core-Collapse Supernova Explosions," arXiv:astro-ph/0510687.

[27] J. M. Bardeen, B. Carter, and S. Hawking, "The Four Laws of Black Hole Mechanics", Commun. Math. Phys. 31, 161 (1973) 